\title{
Desarrollo de audiovisuales bilingües para optimizar el proceso de enseñanza-aprendizaje de estudiantes de Química Analítica
}

\begin{abstract}
Ana Maria Diez-Pascuala ${ }^{a}$, Beatriz Jurado Sanchez ${ }^{a}$, Raquel Lázaro Gutiérrez ${ }^{b}$, Bruno Echauri Galván ${ }^{\mathrm{b}}$, María Jesús Fernández Gil ${ }^{\mathrm{b}}$ y David Fernández Vítores ${ }^{\mathrm{b}}$

aDepartamento de Química Analítica, Química Física e Ingeniería Química (am.diez@uah.es, beatriz.jurado@uah.es) bepartamento de Filología Moderna (raquel.lazaro@uah.es, bruno.echauri@uah.es, mj.fernandezg@uah.es, david.vitores@uah.es). Universidad de Alcalá, Alcalá de Henares, Madrid.
\end{abstract}

\begin{abstract}
This work summarizes the preliminary experience in the development of bilingual audiovisuals as a training resource to improve the teachinglearning process for Analytical Chemistry students. The selection of the technical and practical contents for the video production and the elaboration of a didactic script was performed by teachers belonging to the Analytical Chemistry area. Students of the Degree in Modern Languages and Translation under the supervision of teachers from the Modern Philology Department were in charge of the translation into English of the didactic materials: practical laboratory guidelines and equipment manuals. In addition, work groups were set up among students of Analytical Chemistry and Modern Languages and Translation, and they are developing, using a collaborative pedagogical methodology, the audiovisuals: translation of videotutorial scripts, voice over and subtitling of videotutorials, set up and video editing. The didactic experience has proved to be very positive, allowing the students to acquire and reinforce general and specific competences. The prepared videotutorials will be incorporated into the "Open Education" platform of Alcalá University, and their didactic efficiency will be analyzed by comparing the academic achievement of students who used videotutorials and those who only followed the conventional teaching methods.
\end{abstract}

Keywords: bilingual audiovisuals, teaching-learning process, competences, didactic material, collaborative work

\footnotetext{
Resumen

Este trabajo recoge la experiencia inicial en el desarrollo de audiovisuales bilingües como recurso formativo para mejorar el proceso de enseñanzaaprendizaje de estudiantes de Química Analítica. La selección de los contenidos técnicos y prácticos para la producción de los vídeos y la elaboración del guion didáctico fue realizada por profesores pertenecientes al área de Química Analítica. Estudiantes del Grado en Lenguas Modernas y Traducción bajo la supervisión de profesores del Departamento de Filología
} 
Desarrollo de audiovisuales bilingües para optimizar el proceso de enseñanza-aprendizaje de estudiantes de química analítica

Moderna se encargaron de la traducción al inglés de los materiales didácticos: guiones de prácticas de laboratorio y manuales de equipos. Además, se han formado grupos de trabajo entre estudiantes de Química Analitica y de Lenguas Modernas y Traducción, que están elaborando, por medio de una metodología colaborativa, los videotutoriales: traducción de guiones de videotutoriales, locución y subtitulado de videotutoriales, montaje y edición de videos. La experiencia didáctica está siendo muy positiva, permitiendo que los alumnos adquieran y refuercen competencias generales $y$ especificas. Los videotutoriales se incorporarán a la plataforma "Open Education" de la Universidad de Alcalá, y se analizará su eficiencia didáctica compararando el rendimiento académico de los estudiantes que utilizaron videotutoriales y los que únicamente siguieron los habituales métodos de enseñanza.

Palabras clave: audiovisuales bilingües, proceso de enseñanza-aprendizaje, competencias, material didáctico, trabajo colaborativo

\section{Introducción}

Frecuentemente los estudiantes de grado, especialmente en los primeros cursos, presentan ciertas dificultades para comprender y relacionar determinados conceptos clave que les permitan adquirir las competencias necesarias en las diferentes materias. Esta carencia se ha hecho más evidente en los últimos años, a raíz de la implantación de los nuevos planes de estudio de grado (Pérez-Cañaveras, 2005), dado que, debido al diseño de los mismos, las prácticas de ciertas asignaturas se inician antes de haber completado los temas de teoría, lo que dificulta mucho a los alumnos la compresión de las mismas. Además, muchos estudiantes llegan a la universidad sin haber realizado previamente ninguna práctica de laboratorio, una circunstancia que representa un problema añadido. También hay que tener en cuenta la dificultad intrínseca de los conceptos que se abordan, el poco tiempo disponible para explicarlos en detalle en clase, la imposibilidad de tratar en las sesiones prácticas todos los conceptos explicados en las sesiones de teoría, la dificultad del alumno para entender y asimilar los conceptos al ritmo en que se imparten y la dificultad para distinguir lo que es relevante de lo que no lo es, a pesar de las indicaciones de los profesores, así como el cambio en la forma en que los alumnos esperan que se les comunique la información.

Por otro lado, resulta difícil conseguir una participación activa de los estudiantes en las diferentes actividades que se proponen en la asignatura, así como mantener su interés, principalmente porque los estudiantes no reconocen la aplicación del conocimiento a su campo de estudio. No es factible hacer frente a la totalidad de los problemas arriba mencionados, pero sí es posible abordar la solución de algunos de ellos con el fin de minimizar su influencia en el rendimiento académico del estudiante. En particular, se pueden crear nuevos contenidos didácticos con herramientas que resulten atractivas para el estudiante. En este sentido, el uso de vídeos y juegos tiene un impacto muy positivo en la 
adquisición de conocimiento (Pindado, 2005), y contribuye al desarrollo de las capacidades cognitivas del estudiante, incluida la capacidad de interpretación, el pensamiento crítico y las habilidades para la resolución de problemas (Urquidi-Martín y Tamarit-Aznar, 2015). El uso de vídeos también tiene beneficios en la motivación de los estudiantes (He, 2012), y hace que estos, en lugar de permanecer pasivos como en las clases magistrales, participen activamente en el proceso de aprendizaje (e.g. resolución de problemas, construcción de conocimiento), reforzando así el aprendizaje presencial del alumno y mejorando su rendimiento académico (Rodenas, 2012) Esta herramienta hace más atractivo el aprendizaje y fomenta el autoaprendizaje por su flexibilidad y posibilidad de repetición de actuaciones (es posible parar y revisar los vídeos tantas veces como se quiera). Así, el estudiante tiene un mayor control de su proceso de aprendizaje al poder elegir su propio ritmo de seguimiento y asimilación de contenidos en función de su capacidad y circunstancias personales.

En línea con lo anterior, las tecnologías de la información y de las comunicaciones (TICs) han promovido en los últimos años la aparición de los vídeos tutoriales, sistemas didácticos de autoaprendizaje que muestran al alumno el desarrollo de algún procedimiento o los pasos para realizar una determinada actividad (Diaz-Cintas, 2008; Jordan, 2016). Un videotutorial se caracteriza por su facilidad a la hora de explicar conceptos o procesos complejos de manera visual y clara (Arasasingham, 2011; Casado, 2010).

El uso de tutoriales audiovisuales de corta duración es un recurso formativo muy atractivo para los estudiantes (He, 2012), ampliamente familiarizados con la posibilidad de conseguir información a través de dispositivos electrónicos, tales como ordenadores, smartphones y tabletas. Conocidos también como "píldoras formativas", se trata de pequeñas piezas de contenido audiovisual diseñadas para complementar las estrategias convencionales de formación. Se consigue además una formación más eficaz, atractiva y amena porque posibilita integrar elementos multimedia. La formación a través de vídeos puede impartirse por medio de las siguientes vías: 1) Webinar: es un seminario que se sigue a través de web en el que un profesor transmite sus conocimientos a un conjunto de alumnos. Tiene lugar en tiempo real dando la posibilidad a los alumnos de participar mediante preguntas y sugerencias normalmente a través de un chat. 2) Streaming: una clase o una tutoría sobre un tema determinado que el alumno sigue en vivo. 3) Grabaciones: el vídeo queda grabado y colgado en un servidor con la posibilidad de visualizar o descargar en cualquier momento. Esto le da al alumno una gran libertad horaria y puede ir componiendo sus clases cuando le sea posible en la duración que crea conveniente.

El éxito del vídeo en formación se debe a que posibilita un acceso masivo a nivel mundial con un desarrollo completamente en línea. Ofrece una mayor posibilidad de horarios, adaptación a los formatos e interacción estudiante-profesor o estudiante-estudiante, con un entorno más atractivo y eficaz. Su inclusión en las unidades de aprendizaje que componen los modernos cursos MOOC (Massive Open Online Courses) y que constituyen una de las características fundamentales de este tipo de cursos, ha aumentado su popularidad y su utilización en todo tipo de cursos virtuales (Manotas Salcedo, 2018). 
En la red hay muchas plataformas de cursos MOOC. Agrupadas por materia, modalidad o idioma, entre otras cosas, estas webs en línea permiten acceder a cursos ofertados por diferentes y prestigiosas instituciones y universidades. Sin embargo, hasta la fecha existen pocos cursos que incluyan videotutoriales bilingües, lo cual puede proporcionar un gran valor añadido para estudiantes de habla inglesa e hispana (Gonzalez-Vera, 2017).

Visto el potencial de los videotutoriales bilingües, el presente trabajo busca potenciar su aplicación en la enseñanza universitaria a través de una iniciativa encaminada a diseñar y fomentar su empleo como herramienta de apoyo para el aprendizaje de asignaturas que se imparten en el área de Química Analítica. Se ha recurrido al uso de videotutoriales, entre otras razones, porque la visualización de vídeos online ha crecido enormemente en los últimos años (se estima que se suben a la plataforma YouTube 100 horas de vídeo por minuto), lo que los convierte en un medio de comunicación globalmente aceptado y muy utilizado por el alumnado (Sugimoto, 2013). Los alumnos esperan aprender del mismo modo en que actualmente se comunican y se informan, y les atraen los materiales directos, "sin libros" y "sin profesor". Adaptarse a esto requiere hacer cambios en el proceso de enseñanza-aprendizaje según la forma tradicional.

Asimismo, el trabajo se enmarca dentro de la estrategia de internacionalización que la Universidad de Alcalá está siguiendo en los últimos años. La iniciativa conecta perfectamente con otras emprendidas e impulsadas desde el Vicerrectorado de Relaciones Internacionales, como la elaboración de asignaturas English-friendly o la preparación de asignaturas bilingües en distintas titulaciones para atraer a un mayor número de estudiantes internacionales. Así, los potenciales beneficios del trabajo no se circunscriben solo a sus participantes, sino que pueden redundar en favor de la proyección internacional de la Universidad y de su atractivo como destino para alumnos extranjeros.

Como valor añadido, conviene subrayar que, en este caso, el apoyo a esta estrategia de internacionalización se vehicula a través de la colaboración entre alumnos de distintos grados de la UAH, lo cual potencia las sinergias entre campos de cononcimiento diferentes al tiempo que permite al alumnado poner en prácticas los conocimientos que han ido adquiriendo a lo largo de los distintos cursos de sus correspondientes titulaciones.

\section{Objetivos}

- Utilizar las nuevas tecnologías para facilitar la comprensión de conceptos y el correcto empleo de determinadas técnicas y procedimientos utilizados en asignaturas del área de Química Analítica.

- Emplear medios alternativos de apoyo para la optimización del proceso de enseñanzaaprendizaje bilingüe.

- Transferir conocimientos que permitan a los alumnos comprender y relacionar los conceptos clave de las materias y adquirir las competencias necesarias

- Elaborar materiales didácticos bilingües de manera colaborativa entre docentes de diferentes departamentos.

- Fomentar la colaboración activa entre estudiantes de diferentes grados. 
- Fomentar el trabajo del estudiante fuera del aula y guiarle en su autoaprendizaje.

- Comparar los rendimientos y el grado de adquisición de competencias a través de los videotutoriales con aquellos adquiridos con la explicación tradicional de las clases presenciales y la lectura de material bibliográfico.

- Crear una asignatura accesible a estudiantes extranjeros que potencie la internacionalización de la Universidad de Alcalá y aumente su atractivo como destino de movilidades internacionales.

\section{Desarrollo de la innovación}

En general, las asignaturas del área de Química Analítica incluyen, además de las sesiones teóricas, clases prácticas impartidas en grupos pequeños donde el alumno desarrolla experimentos para aprender a aplicar e interpretar los principios básicos estudiados en las clases teóricas.

Estas prácticas tienen como objetivo iniciar a los estudiantes en la sistemática del proceso analítico y capacitarlos para desarrollar criterios científicos en la evaluación de resultados analíticos. Entre sus competencias específicas se encuentran fomentar el trabajo en equipo asi como conocer y saber usar el instrumental y el aparataje que se utilizan habitualmente en este tipo de laboratorios. Para complementar estas actividades prácticas, se han elaborado una serie de vídeotutoriales bilingües de corta duración. En el desarrollo del trabajo se siguen las siguientes etapas:

- Selección de los contenidos técnicos y prácticos propios de la producción del vídeo.

- Elaboración de un guion didáctico.

- Preparación del videotutorial.

- Difusión del tutorial audiovisual.

- Desarrollo de instrumentos de evaluación de las competencias adquiridas por los alumnos

\subsection{Selección de los contenidos del vídeo}

Los contenidos han sido seleccionados y desarrollados por profesores pertenecientes al área de Química Analítica. Se han priorizado aquellos conceptos, técnicas y procedimientos que presentan mayor dificultad para el aprendizaje por parte de los alumnos. Se elaborado un listado con los contenidos seleccionados.

\subsection{Elaboración de un guion didáctico}

Esta tarea también ha sido realizada por los profesores que imparten asignaturas de Química Analítica. Para ello se han apoyado en los conocimientos aprendidos en el curso de formación del profesorado: "Curso básico de medios audiovisuales para la docencia y el aprendizaje autónomo del alumno. Módulo I: elaboración de guiones y toma de imágenes para realizar videos científicos y tutoriales". Se han seguido los siguientes pasos: 
Desarrollo de audiovisuales bilingües para optimizar el proceso de enseñanza-aprendizaje de estudiantes de química analítica

- Elaboración de una sinopsis, es decir, de un resumen de todo lo que aparecerá en el vídeo, para tener la idea clara de lo que se escribirá luego en el guion.

- Tratamiento del relato. El tratamiento es la historia entera que se muestra en el vídeo escrita en forma de narración

- Elaboración del guion cinematográfico. En él se detalla el contenido de cada toma, de cada escena y de cada secuencia.

- Elaboración del guion técnico. En él se especifican los tipos de planos necesarios para cada escena o secuencia, la duración de los mismos, los movimientos de la cámara, la banda sonora y la planificación que aportará información sobre la duración del vídeo

\subsection{Preparación del vídeotutorial}

-Etapa de preproducción: Se ha elaborado un plan de grabación que incluye las fechas y los tiempos que permiten coordinar los diferentes elementos que intervienen dentro de la grabación. Se han preparado preferentemente vídeos cortos (5-10 minutos).

-Etapa de producción: Siguiendo el plan trazado se han tomado las imágenes y se han grabado los audios. Esta tarea la han realizado fundamentalmente los estudiantes del Grado en Lenguas Modernas y Traducción, en el marco de la asignatura Prácticas Externas $\left(4^{\circ}\right.$ curso, 350 horas) en colaboración con estudiantes de Química Analítica. Las traducciones han sido supervisadas por docentes del Dpto. de Filología Moderna. Esta etapa ha incluido las siguientes actividades:

- Traducción de guiones de videotutoriales

- Locución de videotutoriales

- Subtitulado de videotutoriales

- Montaje y edición de vídeos

-Etapa de post-producción: Se han editado los vídeos científicos con ayuda de programas como AdobePremier, y se han incluido efectos visuales, de sonido, musicalización y animación, corrección y edición de la voz en off, y normalización de la tipografía, colores y diseño en general.

\subsection{Difusión del tutorial audiovisual}

Los vídeos elaborados se difundirán a través de la plataforma MOOC de la Universidad de Alcalá “Open Education”, plataforma de acceso libre que pondrá el material a disposición de cualquier estudiante. Para ello se solicitará al Aula Virtual la creación de un espacio adecuado. Los vídeos serán accesibles a estudiantes tanto españoles como de otros países. El carácter bilingüe de los vídeos será de gran utilidad tanto para los estudiantes españoles que cursan asignaturas universitarias en inglés como para estudiantes extranjeros que cursan sus estudios en España.

Los videotutoriales se acompañarán de:

-material complementario de lectura alojado en la misma plataforma -autoevaluaciones del material audiovisual y del complementario 
-evaluaciones por pares, lo que requiere la realización por parte del alumno de un trabajo (en PDF o audiovisual) y su evaluación por otros 2 o 3 compañeros. Los profesores también revisarán los trabajos creados por los alumnos y les indicarán correcciones y/o sugerencias de mejora. Este método permitirá una comunicación continua aprendiz-docente como base fortalecedora del proceso de enseñanza-aprendizaje.

-material generado por los propios alumnos en la plataforma, consistente en la participación en los correos, foros, blogs y wikis.

Para estudiantes de los primeros cursos de grado, este sistema les ayudará en su dedicación al estudio y su autonomía, a trabajar con un ritmo adecuado que favorezca sus progresos y a adquirir una visión global de la materia.

\subsection{Desarrollo de instrumentos de evaluación de las competencias adquiridas}

Se ha elaborado una rúbrica para evaluar la calidad de los videotutoriales y la utilidad de los mismos para el aprendizaje de los alumnos (Tabla 1).

Tabla 1. Rúbrica desarrollada para evaluar los beneficios de los videotutoriales en el aprendizaje de los estudiantes y las competencias adquiridas por los mismos

\begin{tabular}{|c|c|c|c|c|}
\hline & Muy Bueno & Bueno & Satisfactorio & Insatisfactorio \\
\hline $\begin{array}{l}\text { Calidad de } \\
\text { los video- } \\
\text { tutoriales }\end{array}$ & $\begin{array}{lr}\text { El audio se } \\
\text { escucha } \\
\text { manera clara. Las } \\
\text { imágenes } & \text { son } \\
\text { nítidas. } & \text { Los } \\
\text { locutores } & \text { se } \\
\text { entienden bien y } \\
\text { utilizan palabras } \\
\text { comprensibles. Es } \\
\text { muy atractivo, } \\
\text { dinámico y con la } \\
\text { duración } \\
\text { adecuada }\end{array}$ & $\begin{array}{l}\text { La mayor parte de } \\
\text { los audios } \\
\text { escuchan } \\
\text { claramente. } \\
\text { todas las imágenes } \\
\text { son nítidas. En } \\
\text { general } \\
\text { locutores los } \\
\text { entienden bien, pero } \\
\text { alguna palabra es } \\
\text { incomprensible. Es } \\
\text { bastante atractivo y } \\
\text { dinámico }\end{array}$ & $\begin{array}{l}\text { Algunas partes } \\
\text { del audio y } \\
\text { ciertas imágenes } \\
\text { son poco claras. } \\
\text { La locución es } \\
\text { en su mayoria } \\
\text { comprensible. } \\
\text { Es poco } \\
\text { atractivo y } \\
\text { dinámico. La } \\
\text { duración no es } \\
\text { muy adecuada. }\end{array}$ & 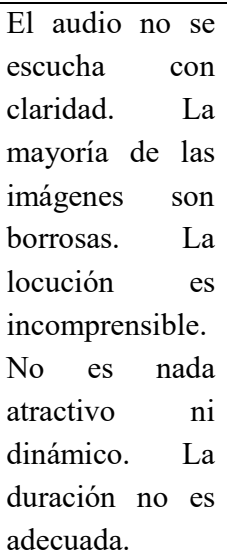 \\
\hline $\begin{array}{l}\text { Desarrollo de } \\
\text { competencias } \\
\text { genéricas y } \\
\text { específicas }\end{array}$ & $\begin{array}{lr}\text { Muestra muchos } \\
\text { ejemplos } \\
\text { promueve el } \\
\text { desarrollo de } \\
\text { competencias: } \\
\text { Capacidad de } \\
\text { autoaprendizaje, } \\
\text { de seleccionar las } \\
\text { técnicas a } \\
\text { adecuadas para la } \\
\text { determinación } \\
\text { analítica de } \\
\text { compuestos y de } \\
\text { valorar la } \\
\text { fiabilidad de los } \\
\text { resultados }\end{array}$ & 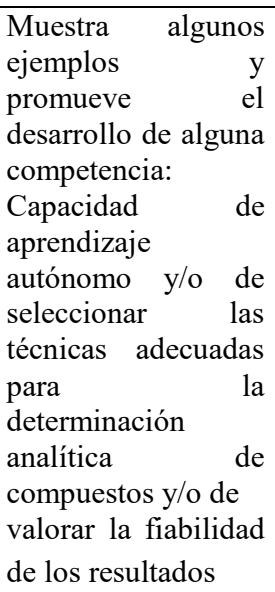 & $\begin{array}{l}\text { Muestra pocos } \\
\text { ejemplos } \\
\text { apenas } \\
\text { promueve el } \\
\text { desarrollo de } \\
\text { competencias: } \\
\text { Capacidad de } \\
\text { autoaprendizaje } \\
\text { o de elegir las } \\
\text { técnicas para la } \\
\text { determinación } \\
\text { de compuestos o } \\
\text { de valorar la } \\
\text { fiabilidad de los } \\
\text { resultados }\end{array}$ & $\begin{array}{lr}\text { Carece } & \text { de } \\
\text { ejemplos y } & \text { no } \\
\text { promueve } & \text { el } \\
\text { desarrollo } & \text { de } \\
\text { ninguna } & \\
\text { competencia. }\end{array}$ \\
\hline
\end{tabular}


Desarrollo de audiovisuales bilingües para optimizar el proceso de enseñanza-aprendizaje de estudiantes de química analítica

\begin{tabular}{|c|c|c|c|c|}
\hline $\begin{array}{l}\text { Relación con } \\
\text { la materia a } \\
\text { estudiar }\end{array}$ & $\begin{array}{l}\text { Emplea la } \\
\text { terminología } \\
\text { propia del tema y } \\
\text { la explica } \\
\text { adecuadamente. } \\
\text { Facilita el } \\
\text { aprendizaje de los } \\
\text { conceptos clave } \\
\text { de la materia. } \\
\text { Ayuda en la } \\
\text { comprensión de } \\
\text { los aspectos más } \\
\text { difíciles. }\end{array}$ & $\begin{array}{l}\text { Emplea la } \\
\text { terminología propia } \\
\text { del tema pero no la } \\
\text { explica } \\
\text { adecuadamente. } \\
\text { Facilita el } \\
\text { aprendizaje de } \\
\text { algunos conceptos } \\
\text { clave de la materia. } \\
\text { Ayuda en la } \\
\text { comprensión de } \\
\text { ciertos aspectos } \\
\text { difíciles. }\end{array}$ & $\begin{array}{lr}\text { Emplea } & \text { un } \\
\text { léxico } & \text { poco } \\
\text { preciso para la } \\
\text { materia. Facilita } \\
\text { el aprendizaje } \\
\text { de } r \text { algún } \\
\text { concepto clave } \\
\text { pero no ayuda } \\
\text { en la } \\
\text { comprensión de } \\
\text { los aspectos más } \\
\text { difíciles. }\end{array}$ & $\begin{array}{lr}\text { No emplea } & \text { la } \\
\text { terminología } & \\
\text { adecuada. } & \text { No } \\
\text { facilita } & \text { el } \\
\text { aprendizaje } & \text { de } \\
\text { ningún concepto } \\
\text { clave de la } \\
\text { material ni } \\
\text { ayuda en la } \\
\text { compressión de } \\
\text { los aspectos más } \\
\text { complejos de } \\
\text { ésta. }\end{array}$ \\
\hline
\end{tabular}

\section{Resultados}

La figura 1 muestra, a modo de ejemplo, el inicio de algunos videotutoriales desarrollados por los alumnos. Estos se han centrado fundamentalmente en la correcta preparación de disoluciones, el uso adecuado de los instrumentos analíticos, tratamiento estadístico de los datos obtenidos y correcta expresión de los resultados.

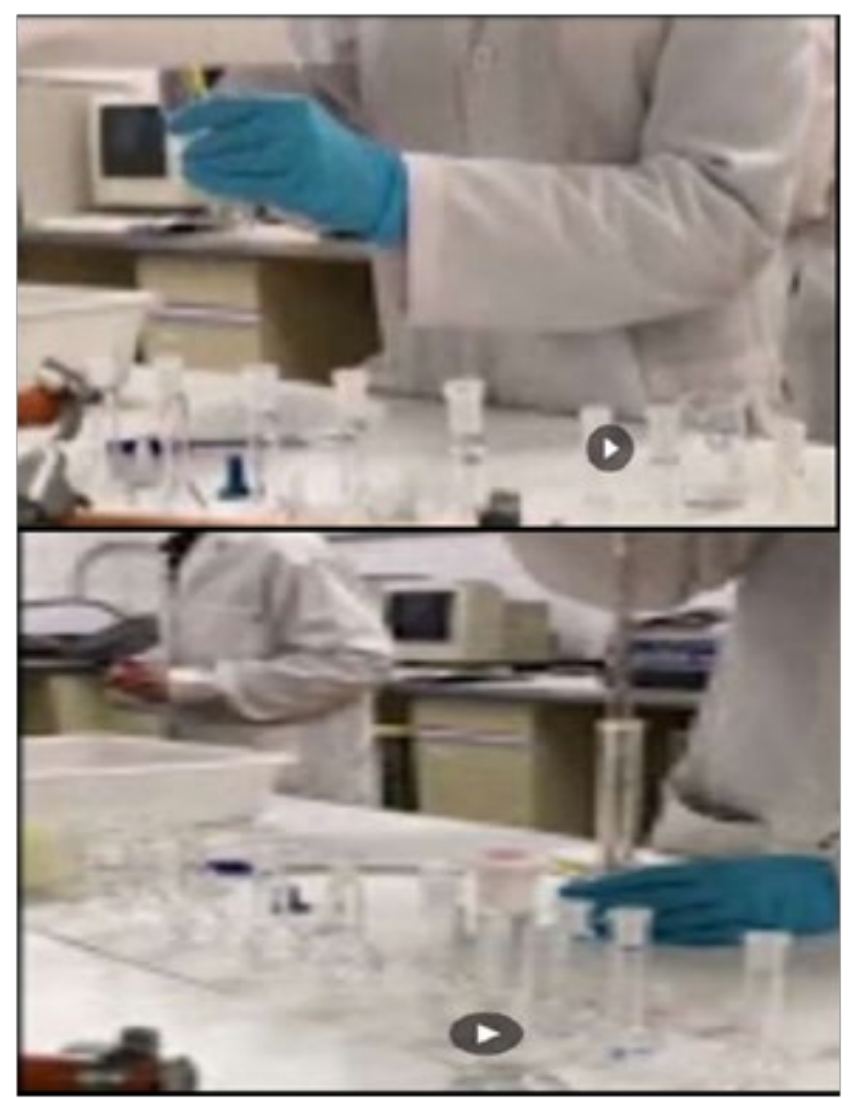

Fig. 1 Capturas de pantalla del inicio de videotutoriales mostrando la preparación de disoluciones 
En lo que a la valoración de la calidad y utilidad de estos materiales se refiere, se ha recogido la opinión de algunos alumnos que han cumplimentado la rúbrica presentada en la Tabla 1. Los resultados obtenidos muestran un grado de satisfacción del alumnado muy alto, siendo todos los aspectos muy buenos para más de un $75 \%$ de ellos. Además, la práctica totalidad de los alumnos manifestaron que recomendarían los vídeos a sus compañeros y que la calidad y duración de los mismos les había parecido adecuada para el aprendizaje de los conceptos más relevantes de la materia. Además de las rúbricas, se incluía un apartado de texto libre para que los alumnos pudieran hacer sugerencias. Entre éstas se repetían el incluir en los videotutoriales un mayor número de ejercicios y sintetizar algunas de las explicaciones de los mismos.

\section{Conclusiones y trabajo futuro}

Este artículo recoge una experiencia de innovación educativa consistente en el empleo de videotutoriales bilingües como herramienta de apoyo para el aprendizaje de los conceptos básicos de Química Analítica. Dicho aprendizaje suele resultar costoso para los alumnos de los primeros cursos de Grado, por lo que un aspecto clave en esta experiencia ha sido la búsqueda de estrategias para que los materiales elaborados resultaran amenos y atractivos, a la vez que útiles. El análisis de las rúbricas cumplimentadas por los alumnos ha arrojado resultados muy prometedores, confirmando que estos han servido para desarrollar competencias generales y específicas de los alumnos.

Por lo que respecta al trabajo futuro, se promoverá la difusión de los videotutoriales desarrollados, incorporándolos a la plataforma "Open Education" de la Universidad de Alcalá. Además, se comparará el rendimiento académico y el grado de adquisición de competencias de los estudiantes que han seguido los videotutoriales con los de alumnos que no hayan seguido los mismos. Se espera que los primeros alcancen un mayor nivel de conocimientos y comprensión para un mejor uso de las técnicas aplicadas en las prácticas.

Tras el análisis de los resultados se procederá a la obtención de conclusiones del beneficio del uso de esta metodología para el proceso de enseñanza-aprendizaje de los estudiantes. A partir de esta experiencia de implementación de videotutoriales bilingües, se propondrán sugerencias para su mejora y aplicación en un futuro a otras materias.

\section{Agradecimientos}

Los autores agradecen el apoyo de la Universidad de Alcalá a través del proyecto de innovación docente UAH/EV983.

\section{Referencias}

ARASASINGHAM, R.D., MARTORELL, I. y MCINTIRE, T. (2011). "Online homework and student achievement in a large enrollment introductory science course" en J. College Sci. Teach., vol. 40, p. 70-79. 
Desarrollo de audiovisuales bilingües para optimizar el proceso de enseñanza-aprendizaje de estudiantes de química analítica

CASADO, N. (2010). Utilización y logros de los audiovisuales en el proceso de enseñanzaaprendizaje. $<$ http://www.uem.es/myfiles/pageposts/jiu/jiu2010/pdf/23c.pdf> [Consulta: 4 de Marzo de 2019]

DIAZ-CINTAS, J (2008). The didactics of audiovisual translation. Amsterdam: John Benhamin Publising.

GONZALEZ-VERA, P. (2017). "La traducción audiovisual como recurso en la enseñanza del inglés específico" en Alejandre Marco, J. L. Buenas prácticas en la docencia universitaria con apoyo de TIC. Experiencias en 2017. Zaragoza: Prensas de la Universidad de Zaragoza. Capítulo 17, p. 157163. ISBN: $978-84-17358-44-0$

HE, Y., SWENSON, S. y LENTS N. (2012). "On line video tutorials increase learning of difficult concepts in an undergraduate Analytical Chemistry course" en J. Chem. Educ., vol. 89, p. 1128-1132.

JORDAN, J. T., BOX, M. C., EGUREN, K. E., PARKER, TH. A., SARALDI-GALLARDO, V. M., WOLFE, M. I. y GALLARDO-WILLIAMS, M. T. (2016). "Effectiveness of student-generated video as a teaching tool for an instrumental technique in the Organic Chemistry laboratory" en J. Chem. Educ., vol. 93, p. 141-145.

MANOTAS SALCEDO, E., PÉREZ RODRÍGUEZ, P. y CONTRERAS, P. (2018). “Análisis de Vídeo-lecciones en MOOC enfocados en la formación pedagógica de docentes en Educación Superior. Un estudio de caso" en REIDOCREA, vol. 7, n 19, p. 248-259.

PÉREZ-CAÑAVERAS, R. M., GÓMEZ-TORRES, M. J. y VIZCAYA MORENO, M. F. (2005). “iInfluyen los cambios de los planes de estudio en el rendimiento académico de los alumnos?” En: II Jornadas de Redes de Investigación en Docencia Universitaria: La configuración del Espacio Europeo de Educación Superior. (9-10.2.2004.Universidad de Alicante, Alicante). 418-433. ISBN 84689-3730-4

PINDADO, J. (2005). "Las posibilidades educativas de los videojuegos. una revisión de los estudios más significativos" en Pixel-Bit. Revista de Medios y Educación, n 26, p. 55-67.

RODENAS, M. (2012). "La utilización de los vídeos tutoriales en educación. Ventajas e inconvenientes. Software gratuito en el mercado" en Revista Digital Sociedad de la Información, $\mathrm{n}^{\circ}$ 33, p. 1-9. <http://www.sociedadelainformacion.com/33/videos.pdf $>$ [Consulta: 5 de marzo de 2019].

SUGIMOTO, C. R., THELWALl, M., LARIVIÈRE, V., TSOU, A., MONGEON, P. y MACALUSO, B. (2013). "Scientists Popularizing Science: Characteristics and Impact of TED Talk Presenters." en PLoS ONE, vol. 8, nº 4, e62403 (8p).

URQUIDI-MARTÍN, A.C. y TAMARIT-AZNAR, C. (2015) "Juegos serios como instrumento facilitador del aprendizaje: evidencia empírica” en Opción, vol. 31, n 3, p. 1201-1220. 